Wind Energ. Sci. Discuss., doi:10.5194/wes-2016-50, 2016

Manuscript under review for journal Wind Energ. Sci.

Published: 22 December 2016

(c) Author(s) 2016. CC-BY 3.0 License.

(c) (i)

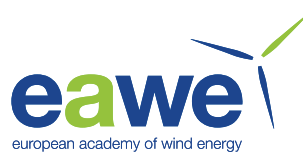

\title{
Methodology for the engineering calculation of flaps on Wind Turbines using BEM codes
}

\author{
Maria Aparicio-Sanchez ${ }^{1}$, Alvaro Gonzalez-Salcedo ${ }^{1}$, Sugoi Gomez-Iradi ${ }^{1}$, and Xabier Munduate ${ }^{1}$ \\ ${ }^{1}$ CENER, Sarriguren, Navarra, 31621, Spain
}

Correspondence to: Maria Aparicio-Sanchez (maparicio@ cener.com)

\begin{abstract}
.
Aeroelastic codes based on Blade Element Momentum theory are the standard used by many wind turbine designers. These codes usually include models and corrections for unsteady aerodynamics, tip and root effect, tower shadow and other effects. In general, this kind of codes does not include models to adequately simulate aerodynamic control devices. This paper presents 5 a method to take into account the unsteady contributions due to the flap motion (based on indicial models) and the spanwise effects (based on circulation theory), in order to simulate flaps on the blades. This method can be included in BEM codes in general and it could also be applied to another kind of control devices. The validation and verification show the accuracy of this method using experimental data for two-dimensional unsteady cases, and CFD for three-dimensional steady and unsteady cases.
\end{abstract}

\section{Introduction}

The classical implementation of the blade element momentum theory is based on steady flow (equilibrium state of wake) and annular ring independence. The proper simulation of the flaps requires an unsteady model for the angle of attack, the flap motion and to include the annular ring dependence due to the spanwise effect of the flaps. The aim of this work is to develop models for the BEM codes in order to correctly simulate the effects of aerodynamic control devices, and the study is focused on trailing edge flaps. The code used for the present study is FASTv8 (Ref.Jonkman et al. (2005), Website), an aeroelastic code developed by NREL and based on the BEM theory.

\section{Approach and methods}

Most of aeroelastic codes consider the unsteady effect of the variation of the angle of attack, and some of them also consider the effect of the flap in the 2D calculation from a steady point of view. These codes can obtain the aerodynamic response with a direct interpolation of static polars through the flap angle. However, this calculation is not complete without the unsteady contribution of the flap motion and the spanwise (three-dimensional) effect generated at the extremes of the flap. 
Wind Energ. Sci. Discuss., doi:10.5194/wes-2016-50, 2016

Manuscript under review for journal Wind Energ. Sci.

Published: 22 December 2016

(c) Author(s) 2016. CC-BY 3.0 License.

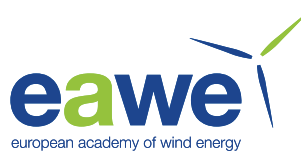

Significant research efforts related to the unsteady contributions of the flaps have been made by Bernhammer (Ref. Bernhammer (2015)), Bæk (Ref. Bæk et al. (2011)), Bergami (Ref. Bergami (2008)) and Gaunaa (Ref. Gaunaa (2006)). Similar modifications in order to simulate dynamic flaps have been adapted to the formulation of FASTv8 and explained in this paper.

The spanwise effects observed in wind turbines have been studied by Pirrung, and a near wake model has been developed 5 in order to reduce the limitations of the BEM codes (Ref. Pirrung et al. (2016a), Pirrung et al. (2016b)), using a coupling between the models (not specifically for flaps). This work is based on the work of Beddoes (Ref. Beddoes (1987)), and the developments of Wang and Coton (Ref. Wang and Coton (1999)) and Madsen (Ref. Madsen and Rasmussen (2004)). There is an specific application for flaps developed by Andersen (Ref. Andersen et al. (2010)). In the present work, the study is focused on the spanwise effect of the flaps. This method is basically based on BEM (with the introduction of vorticity concepts) and leads to lower computational cost.

\subsection{Unsteady method}

The use of smart blade technologies, for example trailing edge flaps, is one of the options to control the loads on wind turbine blades. One of the main objectives is the reduction of fatigue loads and, consequently, this technology must adjust lift quickly and attenuate these loads. It is important to notice that the simulation of the unsteady contribution will be a critical issue for the validity of the flap modelling. Therefore, it is necessary to take into account the current state and also the speed of the changes. Currently, engineering codes usually include models for unsteady aerodynamics: Beddoes-Leishman (Ref. Leishman and Beddoes (July 1989)), ONERA (Ref. Tran et al. (1981)) or Øye (Ref. Øye (1991)).

The oscillations of a trailing edge flap are equivalent to a change in the angle of attack (based on thin airfoil theory, Ref. Leishman (1991), Bisplinghoff et al. (1955)). Therefore, it is possible to use a similar formulation in the calculation of the aerodynamic load coefficients, based on Beddoes-Leishman model (Ref. Leishman and Hariharan (Sept-Oct 1996)).

Considering the flap deflection $(\beta)$, a first order delay is applied to calculate the effective value $\left(\beta_{\text {effn }}\right)$. This delay takes into account the unsteady contribution with a formulation similar to the one used for the angle of attack for the $\mathrm{n}$ time step. $X_{\beta n}$ and $Y_{\beta n}$ are deficiency functions depending on the previous value of the flap deflection $\left(\beta_{n-1}\right)$. The effective value, $\beta_{\text {effn }}$, is then used to get the force coefficients.

The parameters used in the unsteady formulation (i.e. $C_{N \alpha}$, slope of the $2 \mathrm{D}$ normal force coefficient curve and $\alpha_{0}$, zerolift angle of attack) are obtained through a direct interpolation of the input data of the different deflections, using the $\beta_{\text {effn }}$ previously calculated. For instance, the normal coefficient obtained with FAST will have a similar formulation to the calculation without flap, using different values of the parameters. 
Wind Energ. Sci. Discuss., doi:10.5194/wes-2016-50, 2016

Manuscript under review for journal Wind Energ. Sci.

Published: 22 December 2016

(c) Author(s) 2016. CC-BY 3.0 License.

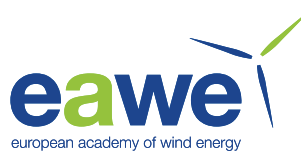

In the tangential coefficient, an additional term is added, in order to include the unsteady effect of the flap deflection.

$C_{T}=C_{N} \cdot\left(\alpha_{e f f}-\frac{\partial C_{N \beta}}{\partial C_{N \alpha}} \cdot\left(\beta_{e f f}-\beta\right)\right)$

The main novelty of this implementation with respect to previous works is the unsteady contribution of $C_{M}$ due to the variation of $\beta$. It depends not only on the variation of $C_{L}$ but also on the variation of the point of application of the resultant $C_{L}$. In the case of flaps, the hysteresis observed in the $C_{M}$ cycles is smoother than the hysteresis of the $C_{L}$ cycles. As this variation is not known using the current methodology, the calculation of $C_{M}$ is interpolated directly with another $\beta_{\text {effn }}$ $\left(\beta_{\text {effCMn }}\right)$, which is defined with a semi-empirical delay.

Currently, the unsteady contribution of the flaps has been included in Aerodyn 14 and in Aerodyn 15 , combined with an improved version of the Beddoes-Leishman model called DYSTOOL (Ref. González et al. (2014)), developed by CENER, which is available in the code FASTv8. The two-dimensional validation have been performed using Aerodyn 15, and the three-dimensional verification with Aerodyn 14.

\subsection{Three-dimensional method}

Pure BEM formulations do not take into account spanwise three-dimensional effects of the flow. However, this type of effects is included using corrections, for example root corrections. A significant difference is observed between the results for a finite flap in a three-dimensional simulation and the two-dimensional approach, therefore the modelling of a flap should include its effect along the blade. Some of the definitions used in other aerodynamic methods are very useful for this new methodology.

First, the definition of circulation and the Kutta-Joukowski theorem is considered. The circulation $(\Gamma)$ for steady conditions is obtained with the lift coefficient calculated by the BEM code.

$\Gamma=\frac{1}{2} \cdot c \cdot V \cdot C_{L}$

The circulation is a macroscopic measure of the rotation for a finite area of the fluid. The circulation could be presented as horseshoes elements (according to the Helmholtz Vortex Theorems) on every strip of the BEM (Figure 1), and the strength of the vorticity emitted from the extremes of each strip is equal to the circulation of that strip. Studying separately these extremes, it is clear that the total vorticity presented at these points is the difference of circulation between the adjacent strips (Figure 2).

$\gamma_{\text {wake }}=\Gamma_{2}-\Gamma_{1}$

The current methodology has been only applied to the extremes of the flap (Figure 3). At the flap extremes, there is a break of the circulation, with a step of the lift coefficient and the emitted vorticity cannot be neglected.

The strength of the vortex filaments is the difference of the circulations in the adjacent regions to the extreme of the flap, whose value is significant. The vortex filaments generate an induced velocity, according to the Biot-Savart Law. Then, the emitted vortex line generates a three-dimensional effect affecting the induced velocity and, consequently, the angle of attack and the force distribution in the closest regions. 
Wind Energ. Sci. Discuss., doi:10.5194/wes-2016-50, 2016

Manuscript under review for journal Wind Energ. Sci.

Published: 22 December 2016

(c) Author(s) 2016. CC-BY 3.0 License.

(c) (i)

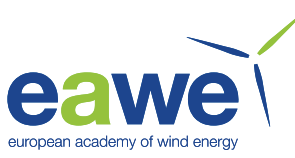

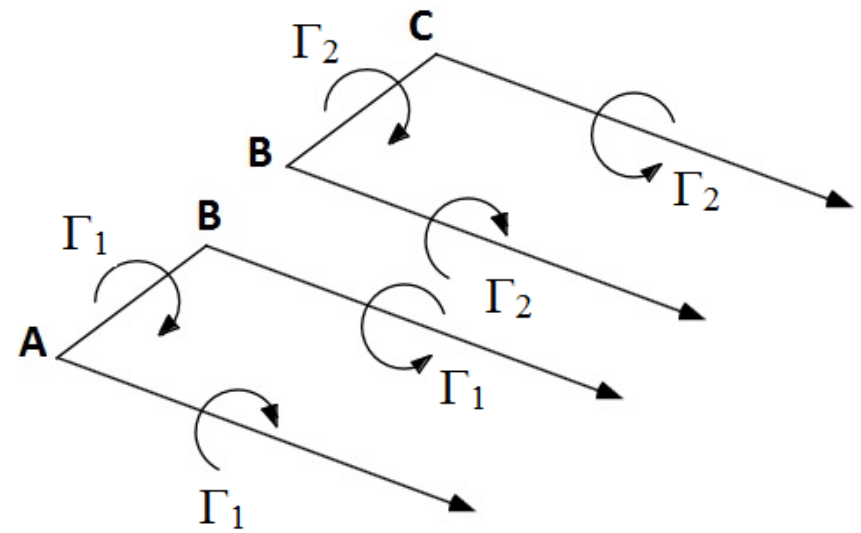

Figure 1. Two adjacent strips of the blade, shown as horseshoes defined by their circulations

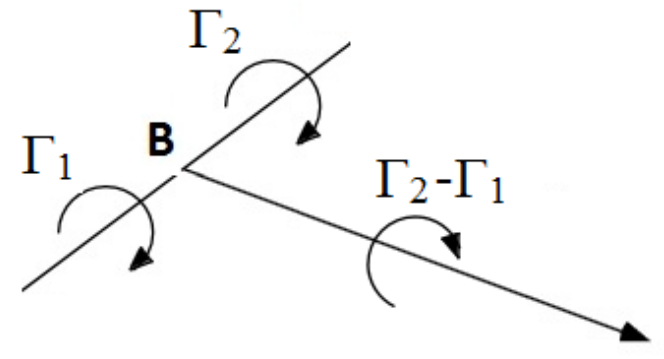

Figure 2. Final result of the emitted line vortex at the common extreme of both strips

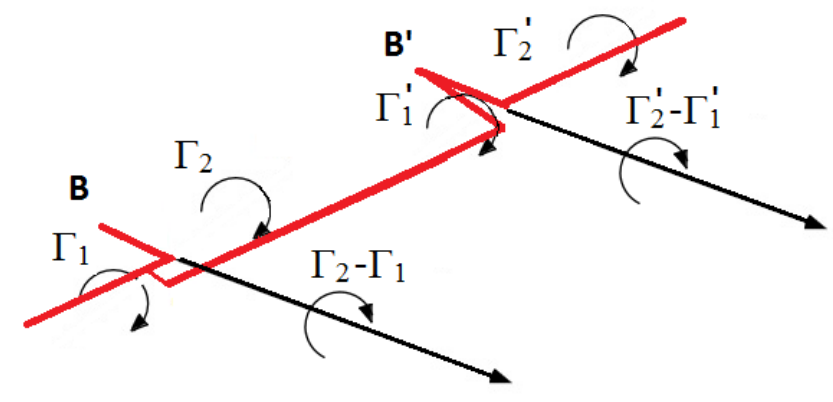

Figure 3. Vortices emitted at the extremes of a flap

The vorticity obtained at the extremes of the flaps is simplified to a straight semi-infinite vortex filament, whose strength is obtained with the following formulation. Where $\gamma$ is the strength of the vortex filament and $\Delta \Gamma$ is the difference of circulation between the adjacent sections and the studied extreme. The parameters $c$ and $V$ are the chord and the relative wind speed of the profile at that section; and $\Delta C_{L}$ the step of the lift coefficient between the adjacent sections to the extreme of the flap.

$5 \gamma=\Delta \Gamma=\frac{1}{2} \cdot c \cdot \Delta\left(V \cdot C_{L}\right)$

The induced velocity is calculated with the Biot-Savart law. The induced velocity at a section is presented as $v_{i}$, and $r$ represents the distance between that section and the extreme of the flap.

$v_{i}=\frac{\gamma}{4 \pi \cdot r}$

In the core region $(r \rightarrow 0)$, the effect of the vorticity is attenuated in order to avoid unrealistic induced velocities when the distance approaches zero. 
Wind Energ. Sci. Discuss., doi:10.5194/wes-2016-50, 2016

Manuscript under review for journal Wind Energ. Sci.

Published: 22 December 2016

(c) Author(s) 2016. CC-BY 3.0 License.

The increment of angle of attack $(\Delta \alpha)$ due to the induced velocity is approximately:

$\Delta \alpha \simeq \frac{v_{i}}{\omega R}$

Where $\omega$ is the rotational speed and $R$ is the radius of the studied section.

$\Delta \alpha \simeq \frac{\Delta \Gamma}{4 \pi \cdot r \cdot \omega R}=\frac{\frac{1}{2} \cdot c \cdot \Delta\left(V \cdot C_{L}\right)}{4 \pi \cdot r \cdot \omega R}$

5 The final equation implemented in the aerodynamic code is the following simplification.

$\Delta \alpha \simeq c \cdot \frac{\Delta C_{L}}{8 \pi \cdot r}$

The filament vortex has been simplified to a straight vortex line with constant strength in order to avoid the additional computational cost of the wake induction calculation. However, the straight vortex can be modified in order to simulate more approximately the helical shape of the wake.

This methodology has also been applied to unsteady cases, neglecting the unsteady effect of the circulation. For an oscillating flap case $(k \approx 0.15)$, this simplification involves deviations close to $6 \%$ in terms of $\Delta \Gamma_{\max }$ and a delay of approximately 0.3 rad.

\section{Preliminary description of the results}

The unsteady two-dimensional validation was performed using experimental data of a flap oscillation (Ref. Leishman and Hariharan (Sept-Oct 1996)). The first case is the oscillation of a $25 \%$ chord flap in a NACA64A006 profile, at Reynolds Number $\approx 2 M$. The amplitude of the flap movement is $\pm 2.5^{\circ}$, with a reduced frequency $k=0.098$ and the angle of attack is $0^{\circ}$. In order to obtain the two-dimensional result using the current aeroelastic code, the simulation for this case considers straight, parked blades, rigid components and effects (tip and root, tower shadow) are disabled. The agreement between the lift and moment coefficients with respect to the experimental data can be observed in figures 4 and 5. Both cycles present similar behaviour to the experimental data, in terms of maximum and minimum values; and the width of the loops.

Another validation case has been studied, using experimental data carried out by CENER (in the DTU -Lyngby Campus, Red Wind Tunnel). It is an oscillating $15 \%$ chord flap on a NACA64418 profile, with a reduced frequency $k=0.100$ at Reynolds Number $0.5 \mathrm{M}$. The amplitude of the flap oscillation is $\pm 5^{\circ}$, and the angle of attack of this case is $0^{\circ}$. The simulation of this case presents the same considerations explained in the previous validation case (straight, parked blades, rigid components and effects are disabled). The agreement of the lift and moment coefficients is shown in the figures 6 and 7, where the $\mathrm{Cl}$ cycle is similar to the experiments, while the $\mathrm{Cm}$ cycle shows similar loop with a slight deviation towards lower values.

Some of the cases of the previously mentioned Wind Tunnel Campaing (carried out by CENER in the DTU -Lyngby Campus, Red Wind Tunnel) include combined motions of the flap and the oscillation of the pitch of the airfoil. The studied airfoil is 
Wind Energ. Sci. Discuss., doi:10.5194/wes-2016-50, 2016 Manuscript under review for journal Wind Energ. Sci.

Published: 22 December 2016

(c) Author(s) 2016. CC-BY 3.0 License.

(c) (i)

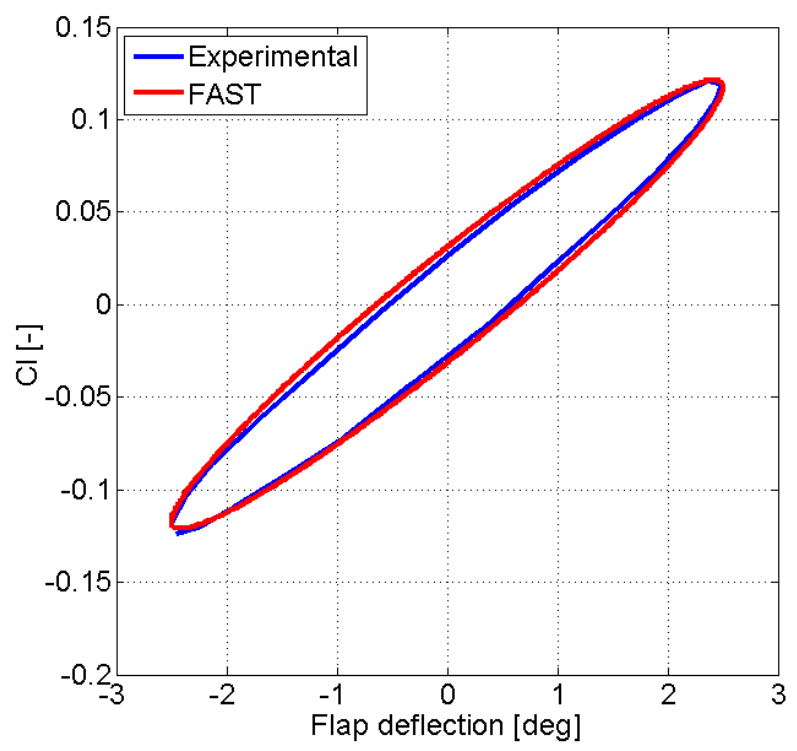

Figure 4. $C_{L}$ for NACA64A006 $k=0.098, \Delta \beta= \pm 2.5^{\circ}, \alpha=0^{\circ}$.

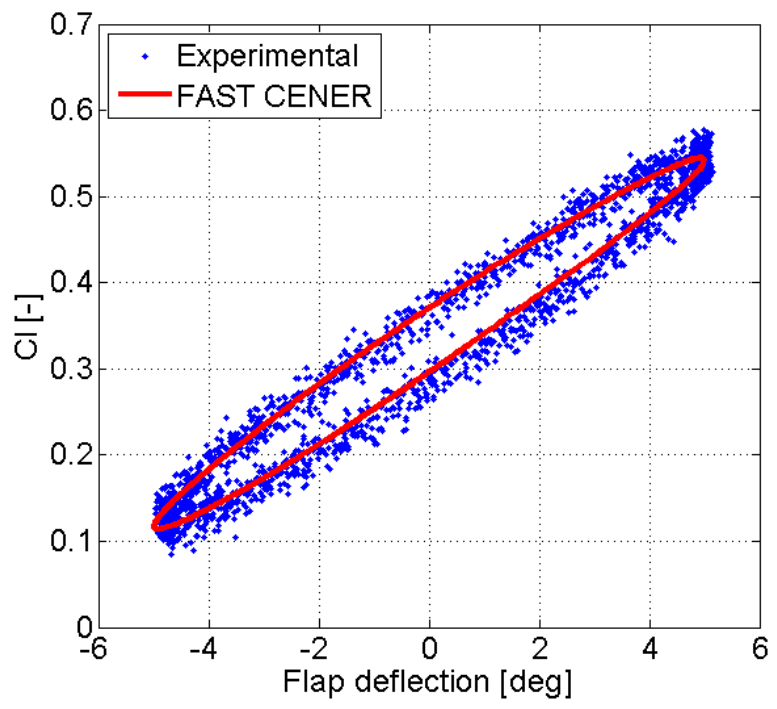

Figure 6. $C_{L}$ for NACA64418 $k=0.100, \Delta \beta= \pm 5.0^{\circ}, \alpha=0^{\circ}$.

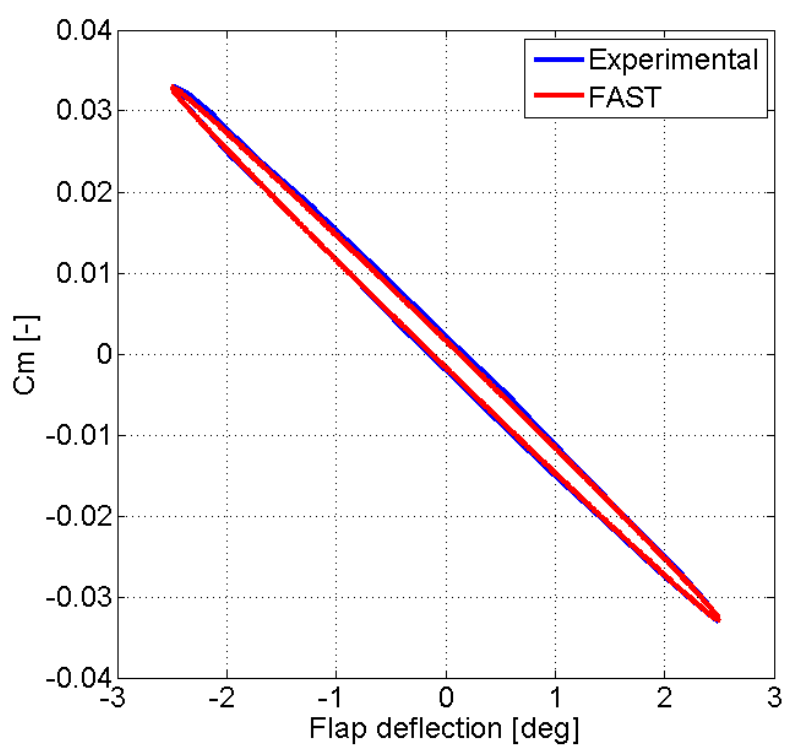

Figure 5. $C_{M}$ for NACA64A006 $k=0.098, \Delta \beta= \pm 2.5^{\circ}, \alpha=0^{\circ}$.

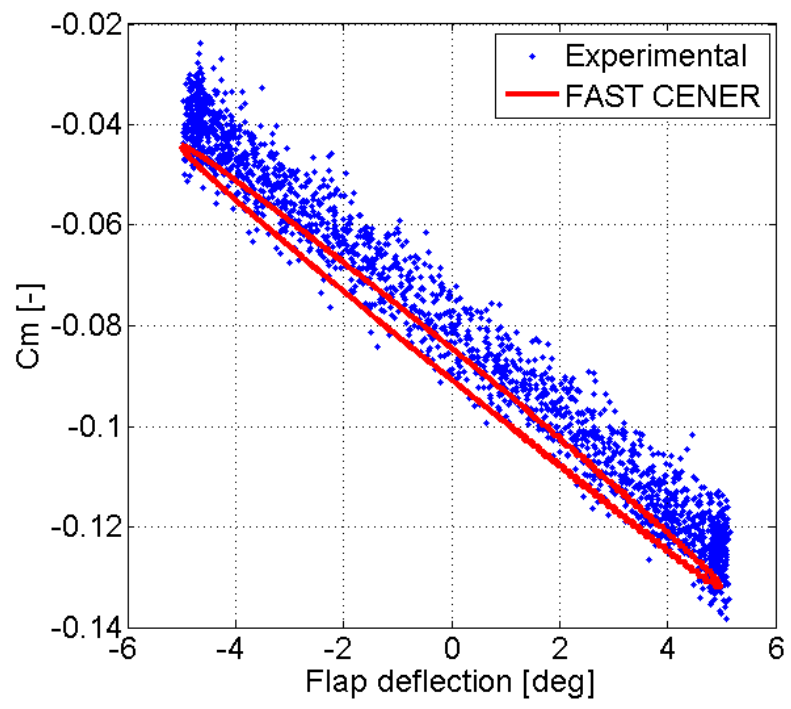

Figure 7. $C_{M}$ for NACA64418 $k=0.100, \Delta \beta= \pm 5.0^{\circ}, \alpha=0^{\circ}$. 
Wind Energ. Sci. Discuss., doi:10.5194/wes-2016-50, 2016

Manuscript under review for journal Wind Energ. Sci.

Published: 22 December 2016

(c) Author(s) 2016. CC-BY 3.0 License.

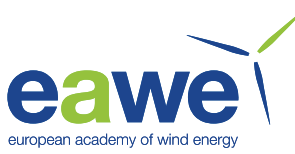

a NACA64418 airfoil with an oscillating 15\% chord flap at Reynolds Number $0.5 \mathrm{M}$. This kind of experiments presents both unsteady effects: the effect of the flap deflection and the oscillation of the pitch angle. The analysis of these cases is really interesting, taking into account that this combined simulation is more realistic than the previous validations, because the combined motions will be observed in the wind turbine and consequently this validation carries an added value. It is important to highlight that the synchronization of the actuators is essential.

The mean angle of attack of the combined cases is $5^{\circ}$, and the amplitude of the oscillation is $\pm 5^{\circ}$, for both cases. The mean flap angle is $0^{\circ}$ and the maximum deflection of the flap is $\pm 5^{\circ}$. The reduced frequency that defines these motions is $k=0.05$. These cases differ in the delay of the angle of attack with respect to the flap deflection $(\delta)$ : the first case presents the same phase for both motions, while the second case shows a delay of $\pi / 2 \mathrm{rad}$. These two validation cases of combined motions of angle of attack and flap deflection has been analysed, with respect to experimental data.
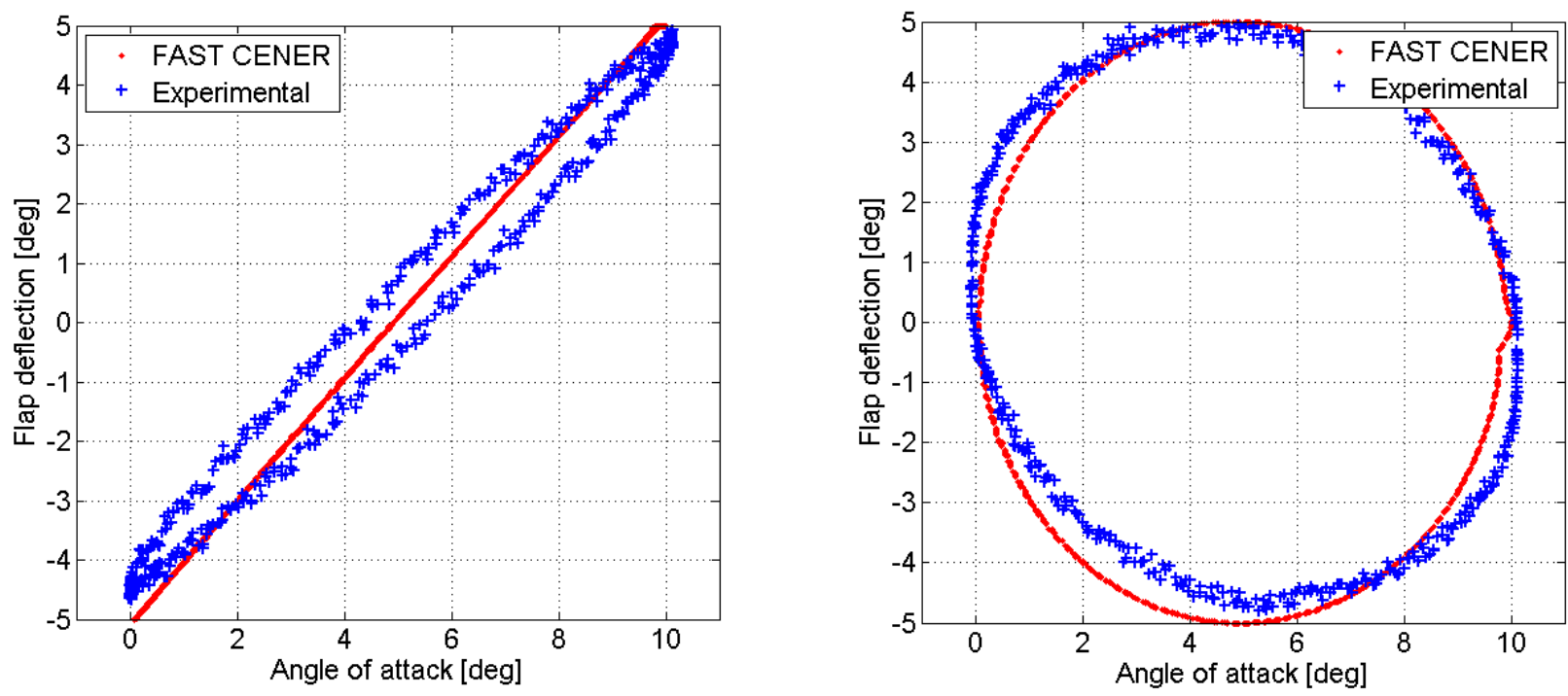

Figure 8. $C_{L}$ with respect to angle of attack for NACA64418 $k=0.05$, Figure 9. $C_{L}$ with respect to angle of attack for NACA64418 $k=0.05$, $\beta_{\text {mean }}=0^{\circ}, \alpha_{\text {mean }}=5^{\circ}, \Delta \beta= \pm 5.0^{\circ}, \Delta \alpha= \pm 5.0^{\circ}, \delta=0^{\circ}$.

$$
\beta_{\text {mean }}=0^{\circ}, \alpha_{\text {mean }}=5^{\circ}, \Delta \beta= \pm 5.0^{\circ}, \Delta \alpha= \pm 5.0^{\circ}, \delta=90^{\circ} \text {. }
$$

First, the agreement of the inputs is shown in figures 8 and 9. The motions of the airfoil are not exactly the same, these small deviations in the delay between the angle of attack and the flap deflection could present differences in terms of force coefficients.

The results obtained for the combined cases can be represented with respect to flap deflection or with respect to angle of attack. Both comparisons have been included in this analysis. Figures 10 and 11 show the cycles obtained for the lift coefficient with respect to the flap angle for both cases. The first one shows a cycle with two clear regions. The loop observed for the lowest flap angles is counterclockwise, while the loop for the highest angles is clockwise. The cycle is narrow and the turning point is slightly different for the experimental case and for the simulation with FAST. This deviation is related to the small 
Wind Energ. Sci. Discuss., doi:10.5194/wes-2016-50, 2016

Manuscript under review for journal Wind Energ. Sci.

Published: 22 December 2016

(c) Author(s) 2016. CC-BY 3.0 License.

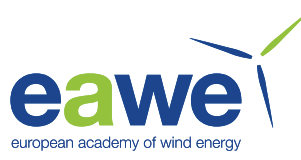

differences observed in the delay between the inputs. The second case presents a clear counterclockwise loop, and the trends observed in the FAST results are similar to the experimental data. The most important deviation of this case is observed for the maximum value of the lift coefficient and it is related to the opposite performance of the unsteady effects of the flap and the angle of attack. This cycle is wider than in the previous comparison, and the cycles with respect to the flap angle are clearly different depending on the delay between the input variables.
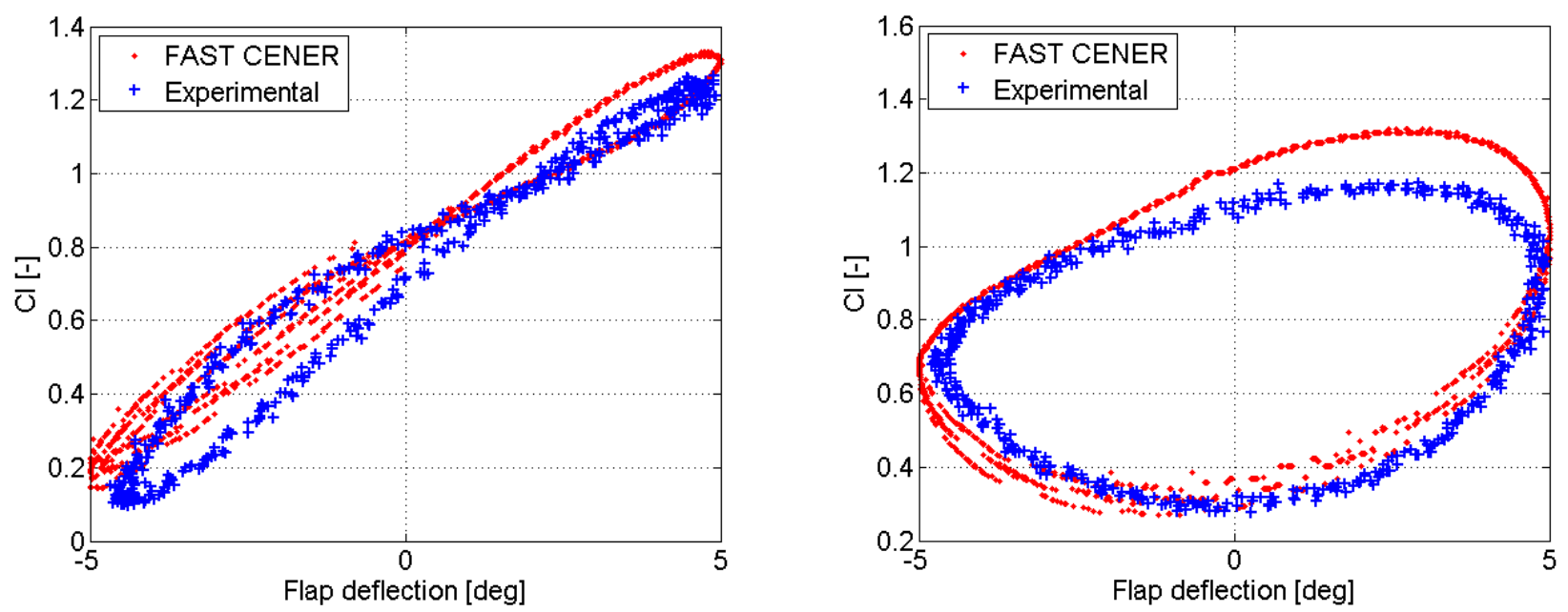

Figure 10. $C_{L}$ with respect to flap deflection for NACA64418 Figure 11. $C_{L}$ with respect to flap deflection for NACA64418 $k=0.05, \beta_{\text {mean }}=0^{\circ}, \alpha_{\text {mean }}=5^{\circ}, \Delta \beta= \pm 5.0^{\circ}, \Delta \alpha= \pm 5.0^{\circ}, \delta=0^{\circ}$. $k=0.05, \beta_{\text {mean }}=0^{\circ}, \alpha_{\text {mean }}=5^{\circ}, \Delta \beta= \pm 5.0^{\circ}, \Delta \alpha= \pm 5.0^{\circ}, \delta=90^{\circ}$.

The comparison of the lift coefficient with respect to the angle of attack is included in figures 12 and 13 for both cases. The shape of the first comparison is quite similar to the comparison with respect to the flap deflection. There are again two different regions and the analysis is similar to the previous comparison of the case. The position of the experimental turning point is very close to the turning point obtained in the simulation, which demonstrates that the differences observed in the previous comparison of the case (with respect to the flap deflection) is due to the delay observed between the flap motion and the pitch oscillation. The second case presents a reasonable good agreement in the clockwise loop. The most significant difference in this case is observed for the maximum lift coefficient (previously mentioned in the comparison with respect to the flap oscillation).

After the validation of the unsteady method implemented, a verification of the three-dimensional method for the flap integration is presented. The accuracy of this new methodology is shown in figures 14 and 15 for a rotating case using the INNWIND.EU rotor without tilt, precone and tower shadow $\left(\omega=8.97 \mathrm{rpm}\right.$, pitch $\left.=4.97^{\circ}\right)$ and a deformable static $10 \% \mathrm{c}$ flap, centred at $75 \% R$, width $14 \% R$ and $\beta=10^{\circ}$ (where $R$ is the distance between the tip and the axis of the wind turbine). These figures show the comparison with respect to CFD results (Ref. Gomez-Iradi (2009)). The results obtained using FAST without the spanwise effects of the flaps have also been included. The increment of the $C_{N}$ due to the effect of the flap is very similar for both codes along the blade, while the $C_{T}$ values present higher differences, related to differences of the static polars. However, the $C_{T}$ trend of the CFD results is clearly captured in the sections close to the extremes of the flap. 
Wind Energ. Sci. Discuss., doi:10.5194/wes-2016-50, 2016

Manuscript under review for journal Wind Energ. Sci.

Published: 22 December 2016

(c) Author(s) 2016. CC-BY 3.0 License.

\section{(c) (1)}

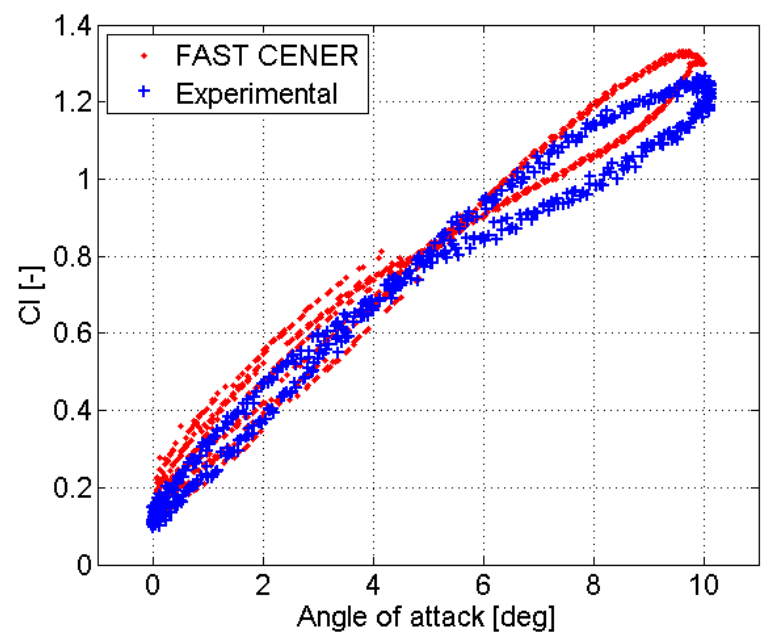

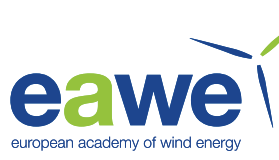

WIND

ENERGY

SCIENCE

DISCUSSIONS

Figure 12. $C_{L}$ with respect to angle of attack for NACA64418 Figure 13. $C_{L}$ with respect to angle of attack for NACA64418 $k=0.05, \beta_{\text {mean }}=0^{\circ}, \alpha_{\text {mean }}=5^{\circ}, \Delta \beta= \pm 5.0^{\circ}, \Delta \alpha= \pm 5.0^{\circ}, \delta=0^{\circ}$.

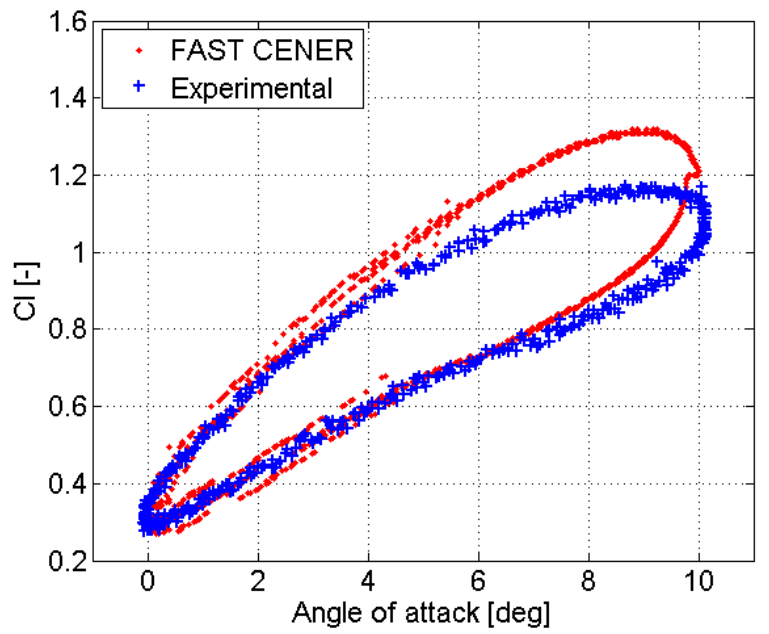
$h=0.05, \beta_{\text {mean }}=0^{\circ}, \alpha_{\text {mean }}=5^{\circ}, \Delta \beta= \pm 5.0^{\circ}, \Delta \alpha= \pm 5.0^{\circ}, \delta=0^{\circ}$. $k=0.05, \beta_{\text {mean }}=0^{\circ}, \alpha_{\text {mean }}=5^{\circ}, \Delta \beta= \pm 5.0^{\circ}, \Delta \alpha= \pm 5.0^{\circ}, \delta=90^{\circ}$.
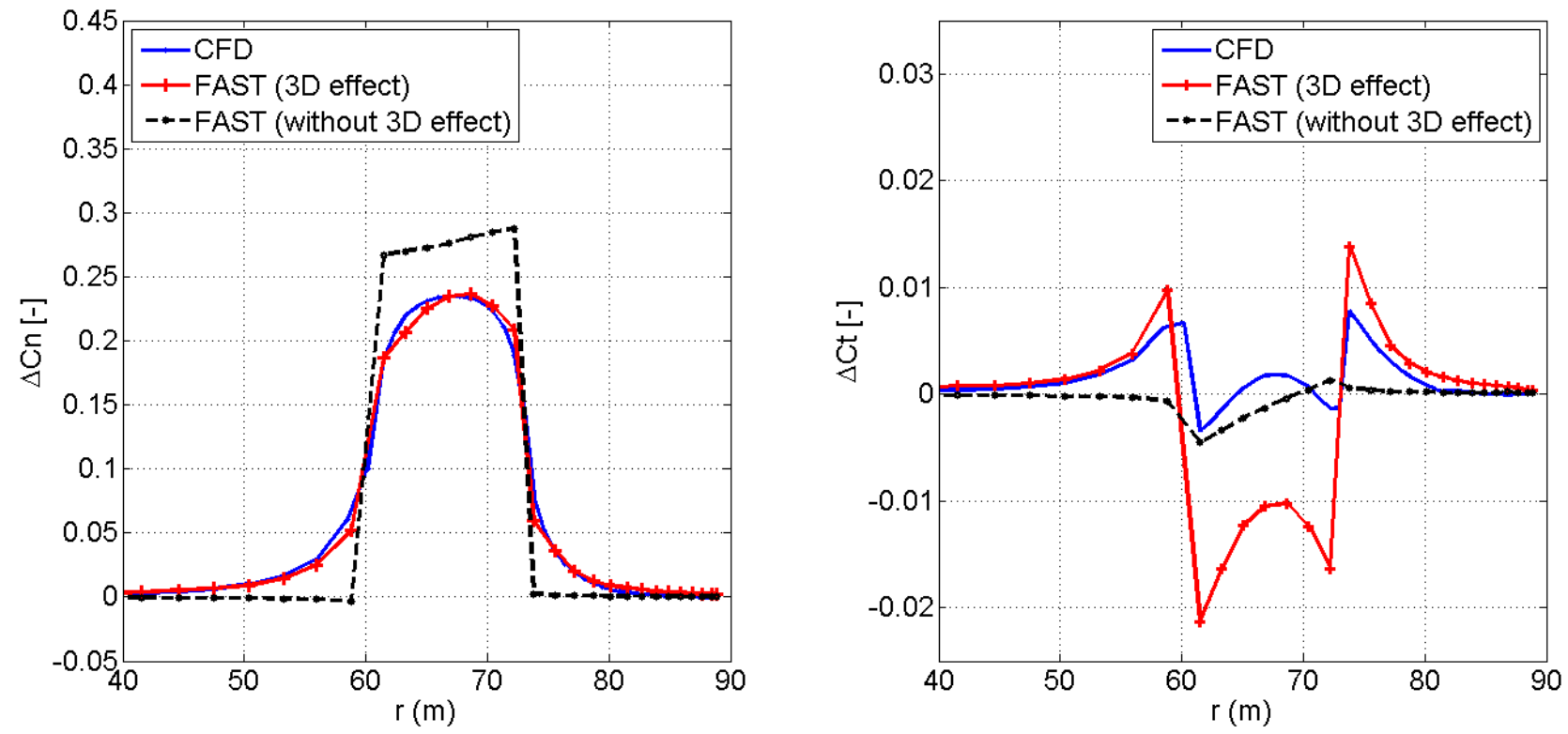

Figure 14. $\Delta C_{N}$ for the INNWIND.EU rotor and $\beta=10^{\circ}$ with re- Figure 15. $\Delta C_{T}$ for the INNWIND.EU rotor and $\beta=10^{\circ}$ with respect spect to the blade without flap. to the blade without flap.

The second case for the three-dimensional validation has been performed with a comparison between different aerodynamic codes, studied within the AVATAR Project (Ref. González et al. (2016)). This case simulates the AVATAR rotor without tilt, 
Wind Energ. Sci. Discuss., doi:10.5194/wes-2016-50, 2016

Manuscript under review for journal Wind Energ. Sci.

Published: 22 December 2016

(c) Author(s) 2016. CC-BY 3.0 License.

\section{(c) (i)}

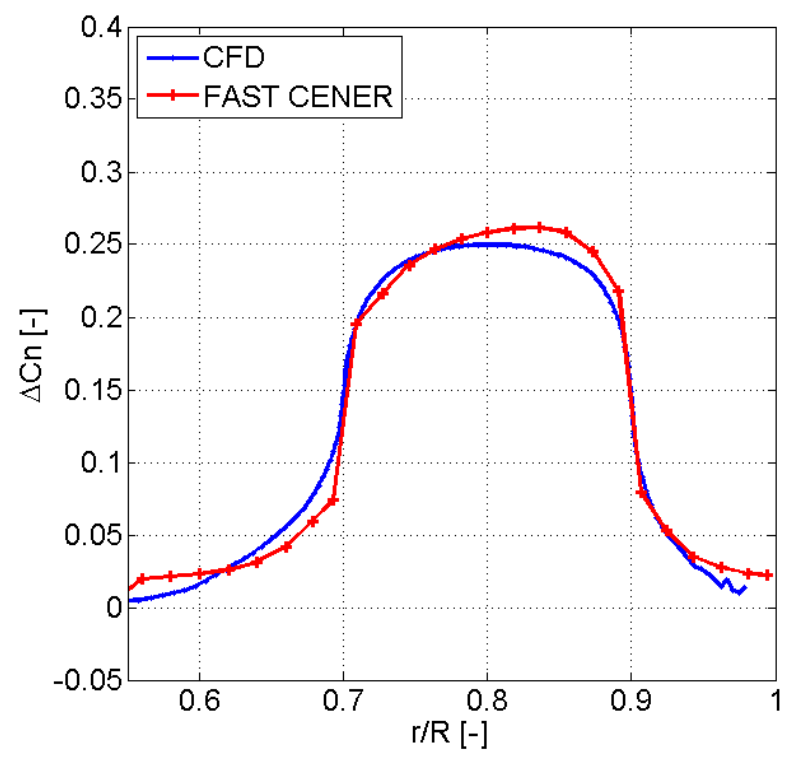

Figure 16. $\Delta C_{N}$ for the AVATAR rotor and $\beta=10^{\circ}$ with respect to Figure 17. $\Delta C_{T}$ for the AVATAR rotor and $\beta=10^{\circ}$ with respect to the blade without flap.

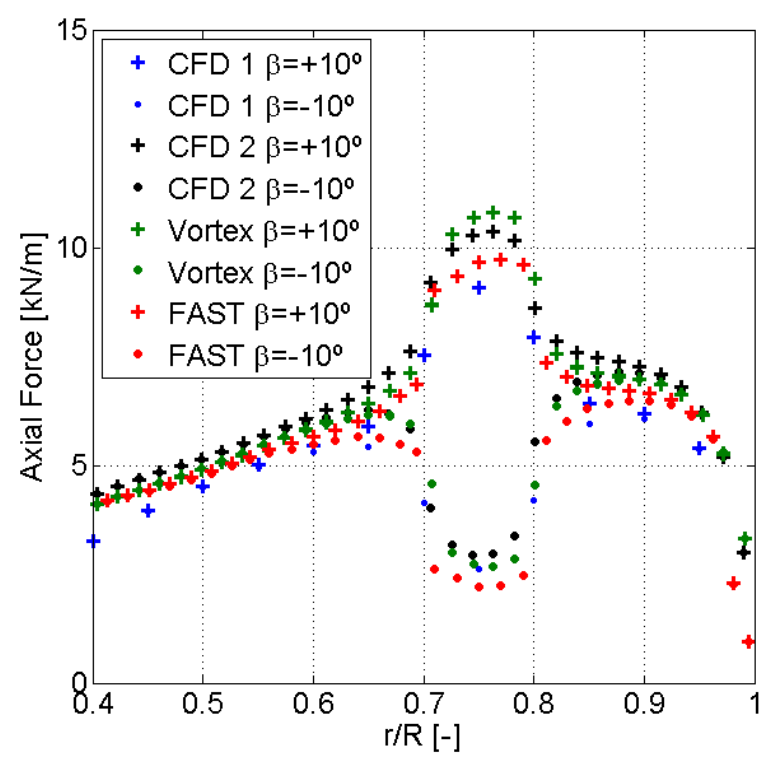

Figure 18. $C_{N}$ for the AVATAR rotor, unsteady case.

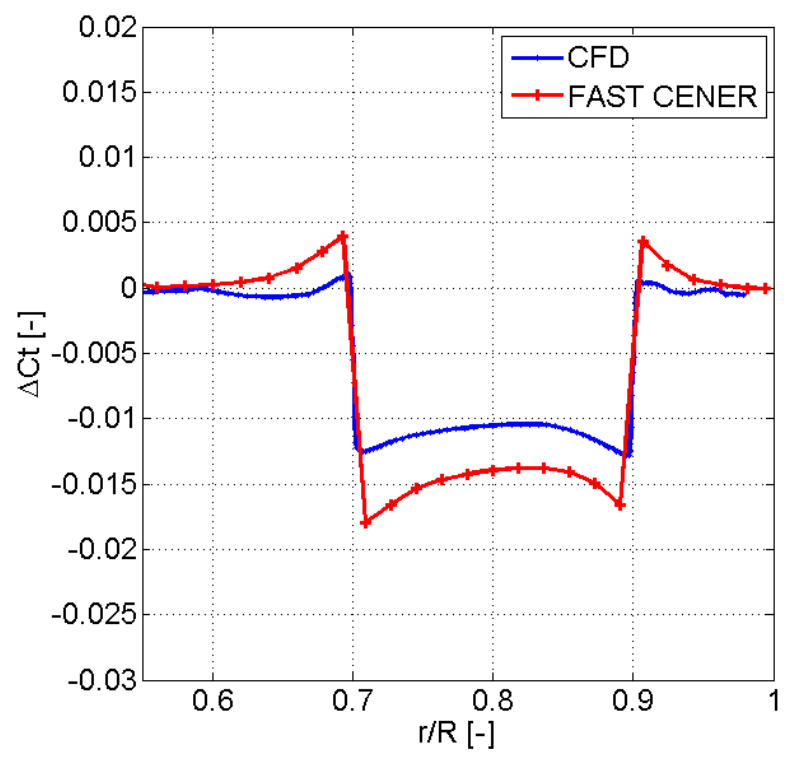

the blade without flap.

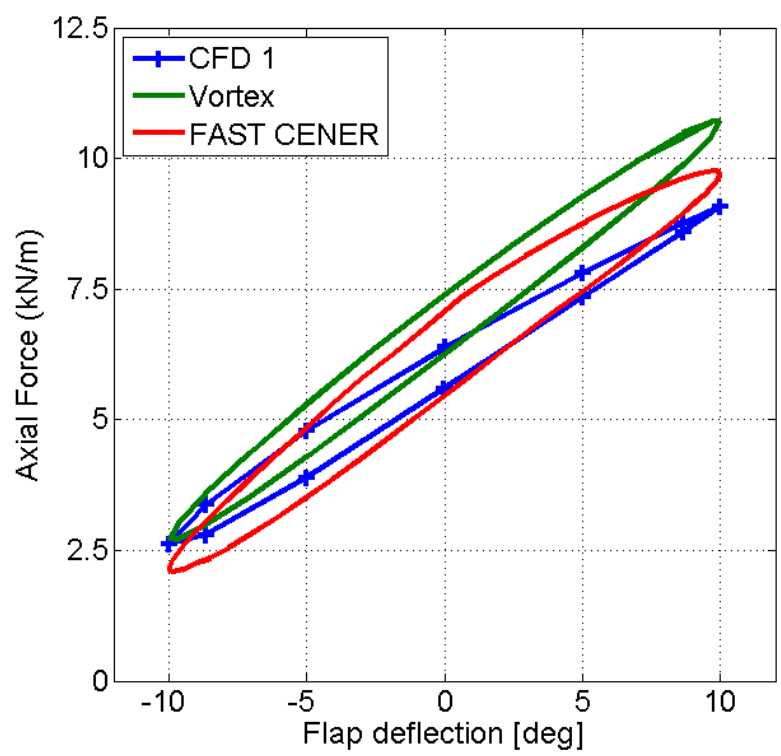

Figure 19. $C_{N}$ at $75 \% R$ for the AVATAR rotor, unsteady case. 
Wind Energ. Sci. Discuss., doi:10.5194/wes-2016-50, 2016

Manuscript under review for journal Wind Energ. Sci.

Published: 22 December 2016

(c) Author(s) 2016. CC-BY 3.0 License.

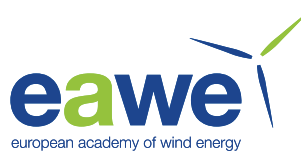

precone and tower shadow $\left(\omega=9.6 \mathrm{rpm}\right.$, pitch $\left.=2.06^{\circ}\right)$ with a deformable steady $10 \%$ c flap (centred at $80 \% R$, width $10 \% R$ and $\beta=10^{\circ}$ ). The figures 16 and 17 present the results obtained with respect to the case without flap for the normal coefficient and the tangential coefficient. The spanwise effects of the flap obtained by FAST are similar to the effects observed in the CFD code for both coefficients.

5 Finally, an unsteady three-dimensional case was performed with the modified aeroelastic code (Figures 18 and 19) within the European Project AVATAR (Ref. Ferreira et al. (2015)). This case simulates the AVATAR rotor without tilt, precone and tower shadow $\left(\omega=9.6 \mathrm{rpm}\right.$, pitch $\left.=2.06^{\circ}\right)$ with an oscillating $10 \% \mathrm{c}$ flap $\left(f_{\beta}=0.96 \mathrm{~Hz}\right.$, centred at $75 \% R$, width $10 \% R$ and $\Delta \beta= \pm 10^{\circ}$ ). It is compared to the results obtained with a vortex method and with two different CFD codes. Figure 18 shows the axial force distribution along the blade for the maximum and the minimum flap deployments and presents similar results of the force along the blade compared to the rest of the codes. In general, the force obtained with FAST is slightly lower than for the rest of the codes. This deviation is related to a difference of the result for the case without flap that could be due to small differences in the static polars. Figure 19 presents the comparison at the mid flap section, and it can be observed a similar slope with respect to the vortex result. The maximum and minimum values present the same deviation previously described.

\section{Conclusions}

This paper demonstrates the possibility to properly simulate flaps in wind turbine blades with a BEM code. The method developed has been presented, including the theories related to this development and the validation (in this case, the aeroelastic code used is FASTv8, Ref. Jonkman et al. (2005), Website). The validation and verification show the accuracy of this method using experimental data for two-dimensional unsteady cases, and for three-dimensional steady and unsteady cases. The results of the validation are very encouraging and present similar trends to CFD results and experimental data.

It is also important to highlight that one of the main advantages presented by the BEM codes with respect to CFD or vortex methods is the computational cost required for the simulations, which is maintained in the same order with the current modifications.

The implementation of the spanwise 3D effect detailed in this work can also be applied to the analysis of blades with abrupt changes of the geometry (i.e. transition between different profiles, important step of the twist or chord and others).

The limitations of this methodology are going to be analyse in future validations. The suitability of this method (including the unsteady method) for another type of aerodynamic control device are going to be studied in future works (i.e. vortex generators, leading edge flaps).

Acknowledgements. The AVATAR European Project has consented the comparison of some of the results previously published in the deliverables.

This Project has received funding from the European Union's Seventh Framework Programme for reseach, technological development and demonstration, under call identifiers no. FP7-ENERGY-2013-1 (Grant Agreement no. 608396). 
Wind Energ. Sci. Discuss., doi:10.5194/wes-2016-50, 2016

Manuscript under review for journal Wind Energ. Sci.

Published: 22 December 2016

(c) Author(s) 2016. CC-BY 3.0 License.

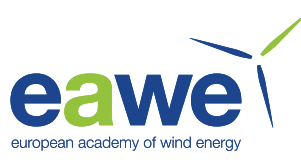

\section{References}

Andersen, P. et al.: A near wake model for deformable trailing edge flaps implemented the in multi body aero-servo-elastic code HAWC2, EWEC 2010 Proceedings online. European Wind Energy Association (EWEA), 2010.

Bæk, P. et al.: A modified dynamic stall model for airfoils with active aerodynamic devices, Wind Energy, 2011.

5 Beddoes, T. S.: A near wake dynamic model, Aerodynamics and Aeroacoustics National Specialist Meeting. Papers and Discussion, 1987.

Bergami, L.: Aeroservoelastic Stability of a 2D airfoil section equipped with a trailing edge flap, Technical Report 1663, Ris $\varnothing$ National Laboratory, 2008.

Bernhammer, L. O.: Smart Wind Turbine: analysis and autonomous flap, PhD at TU Delft, 2015.

Bisplinghoff, R. L. et al.: Aeroelasticity, 1955.

10 Ferreira, C. et al.: Task 3.2 : Development of aerodynamic codes for modelling of flow devices on aerofoils and rotors, AVATAR Project, 2015.

Gaunaa, M.: Unsteady 2D potential-flow forces on a thin variable geometry airfoil undergoing arbitrary motion, Technical Report 1478, Ris $\varnothing$ National Laboratory, 2006.

Gomez-Iradi, S.: CFD for Horizontal Axis Wind turbines, PhD at University of Liverpool, 2009.

15 González, A. et al.: Validation of DYSTOOL for unsteady aerodynamic modeling of 2D airfoils, Proceedings of the Science of Making Torque from Wind, 2014.

González, A. et al.: Deliverable 3.3 : Results of a parametric study of flow devices, guidelines for design, AVATAR Project, 2016.

Jonkman, J. M. et al.: FAST User's Guide, Technical Report NREL/EL-500-38230 National Renewable Energy Laboratory, 2005.

Leishman, J. G.: Unsteady Lift of a Flapped Airfoil by Indicial Concepts, Technical Report, Department of Fluid Mechanics, Technical University of Denmark, 1991.

Leishman, J. G. and Beddoes, T. S.: A Semi-Empirical Model for Dynamic Stall, Journal of the American Helicopter Society, Vol. 34, No. 3, July 1989.

Leishman, J. G. and Hariharan, N.: Unsteady aerodynamics of a flapped airfoil in subsonic flow by indicial concepts, Journal of Aircraft, Vol. 33, No. 5, Sept-Oct 1996.

Madsen, H. and Rasmussen, F.: A Near Wake Model for Trailing Vorticity Compared with the Blade Element Momentum Theory, Wind Energy, 2004.

Øye, S.: Dynamic stall simulated as time lag of separation, Technical Report, Department of Fluid Mechanics, Technical University of Denmark, 1991.

Pirrung, G. et al.: A coupled near and far wake model for wind turbine aerodynamics, Wind Energy, $2016 \mathrm{a}$.

30 Pirrung, G. et al.: Comparison of a Coupled Near and Far Wake Model With a Free Wake Vortex Code, Wind Energy Science, 2016b.

Tran, C. T. et al.: Semi-Empirical Model for the Dynamic Stall of Airfoils in View of the Application to the Calculation of Responses of a Helicopter Blade in Forward Flight, Vertica, Vol.5, 1981.

Wang, T. and Coton, F. N.: A modified near wake dynamic model for rotor analysis, The aeronautical journal, 1999.

Website, F.: FASTv8 Website, https://nwtc.nrel.gov/FAST8. 